\title{
Miranda
}

Revue pluridisciplinaire du monde anglophone /

Multidisciplinary peer-reviewed journal on the English-

speaking world

9 | 2014

Coincidences / Circulating towards and across the British Isles

\section{« It's about being connected »: il est sept heures, New York s'éveille: Let the Great World Spin de Colum McCann ou la coïncidence entre un homme et une ville}

\section{Sophie Vallas}

\section{CpenEdition}

Journals

Édition électronique

URL : http://journals.openedition.org/miranda/6086

DOI : $10.4000 /$ miranda.6086

ISSN : 2108-6559

Éditeur

Université Toulouse - Jean Jaurès

Référence électronique

Sophie Vallas, « «It's about being connected »: il est sept heures, New York s'éveille: Let the Great World Spin de Colum McCann ou la coïncidence entre un homme et une ville », Miranda [En ligne], 9| 2014, mis en ligne le 03 mars 2014, consulté le 16 février 2021. URL : http://journals.openedition.org/ miranda/6086 ; DOI : https://doi.org/10.4000/miranda.6086

Ce document a été généré automatiquement le 16 février 2021. 


\title{
«It's about being connected »: il est sept heures, New York s'éveille: Let the Great World Spin de Colum McCann ou la coïncidence entre un homme et une ville
}

\author{
Sophie Vallas
}

Dans la nuit du 6 au 7 août 1974, Philippe Petit tendit son fil de funambule entre les deux tours du World Trade Center pour une promenade de 45 minutes à 450 mètres de haut au-dessus de la pointe sud de Manhattan. Ces pas aériens qui relièrent les deux tours à peine terminées, ces petits sauts accomplis dans un ciel brumeux, ce corps qui s'étendit sur un câble flottant accomplirent un double exploit. Un homme à la silhouette elfique fit lever le jour depuis le toit du monde, tandis que sa marche improbable et gracieuse fit entrer ces deux bâtiments mal aimés des New-Yorkais dans l'histoire de la ville : le fil du funambule avait réussi à attacher viscéralement les tours orphelines au corps boudeur d'un New York qui n'avait pas l'impression de les avoir conçues. "A stroke of genius » (248), estime le juge Soderberg qui, dans le roman de Colum McCann, va devoir trouver une sentence pour le lutin accoucheur qui comparaît devant lui quelques heures après son exploit: dans cette ville qui n'a pas d'histoire et qui n'éprouve aucun intérêt pour l'histoire, songe-t-il, le funambule a accompli un acte performatif. Au sommet des deux tours les plus arrogantes du monde, son corps a coïncidé avec la ville et s'est fait monument fugace : «He had made himself into a statue, but a perfect New York one, a temporary one, up in the air, high above the city». Et ce corps audacieux, comprend le juge, a magistralement signé une œuvre jusque-là ignorée, deux tours inélégantes qui n'avaient pas encore trouvé leur place dans le paysage urbain.

2 Lorsque Colum McCann entreprend l'écriture de Let the Great World Spin, paru en 2009, le skyline au sud de Manhattan est amputé des tours jumelles et les New-Yorkais en 
ressentent désormais l'absence dans leur chair. La performance de Philippe Petit a été exhumée des souvenirs nostalgiques des années 70 , un peu comme on évoque la bonne fée qui s'est autrefois penchée sur un berceau désormais déshérité : un documentaire inspiré (James Marsh, 2008) en raconte l'histoire et l'exécution rocambolesques. Cette performance se trouve également au cœur du roman de McCann qui s'organise littéralement autour de cette petite heure qui ébranla New York, puisque tous les personnages sont tantôt témoins émerveillés, indifférents ou outragés de la scène, tantôt réceptionnaires d'un récit de l'événement. Lorsque le funambule, qui reste anonyme dans le roman de McCann, regarde les autorités new-yorkaises retirer son câble après son numéro, il le voit s'affiner tout doucement avant de disparaître tout à fait: la ligne est, en effet, démontée dans l'ordre inverse dans lequel elle avait été génialement et péniblement installée - le câble est tiré par une équipe sur l'une des tours tandis qu'une autre équipe, en face, l'empêche de tomber dans le vide grâce à la chaîne à laquelle il est attaché, suivie d'une corde et enfin d'un fil de pêche invisible qui, au début du processus, avait été lancé d'une tour à l'autre au moyen d'un arc :

[...] it was being hauled in, slowly, carefully, attached to a chain, to a rope, to a fishing line. It was like watching a child's Etch a Sketch as the sky shook itself out: the line kept disappearing pixel by pixel. Eventually there would be nothing left there at all, just the breeze. (243)

3 Plus de 25 ans plus tard, McCann reprend «l'ardoise magique » et trace à nouveau le câble torsadé qui permit un jour à un homme de marcher au sommet du monde et à tant d'Américains, les yeux fixés dans les airs, de sentir New York sous leurs pieds. Let the Great World Spin joue ainsi sur la coïncidence à plus d'un titre, comme s'il déclinait différents sens du terme: autour d'un spectacle inattendu, des vies vont en effet fortuitement se croiser et des regards converger; des situations appartenant à différentes intrigues vont se produire de façon simultanée tandis que, sur la carte de New York, des lignes vont se répondre et se superposer.

\section{Un câble tendu entre 1974 et 2001 : roman choral et roman reflet}

4 Les 60 mètres d'acier et de nuages parcourus par le funambule ne sont, en effet, que l'une des lignes que trace le roman: sur sa carte de New York, McCann en place d'autres sur lesquelles il va faire évoluer ses personnages, comme si ceux-ci étaient inspirés par la marche de l'artiste, comme s'ils faisaient écho à ses pas précautionneux sur leurs pauvres câbles terrestres. Au fil du roman, le lecteur est amené à comprendre que ce câble artificiellement tendu au cœur de la ville comme du roman n'est qu'un des éléments d'une structure complexe qui va voir s'entrecroiser de multiples fils. Une scène, en particulier, suggère poétiquement ce principe organisationnel choisi par McCann: le funambule a tendu une corde entre sa fenêtre et l'échelle d'incendie de l'immeuble d'en face pour aller passer la nuit chez une femme. Lorsqu'il reprend son chemin de chanvre, il a la surprise de le retrouver décoré à la mode new-yorkaise: « [...] when he emerged he found that some kids had tossed shoes up on the wire, a city custom, the laces tied together » (239). Autant de lacets à cheval sur la corde, autant de points semés sur la ligne de vie d'un homme qui, pendant quelques instants magiques, parviendra à lier sa trajectoire à la majesté d'une ville, comme le formulera l'un des personnages au sujet de cette traversée entre les deux tours jumelles: "The 
intersection of a man with the city, the abruptly reformed, the newly appropriated public space, the city as art " (103). Le mot «intersection » suggère ici deux sens différents : la coïncidence magistrale de l'artiste et de la ville, certes, mais l'image des paires de chaussures suspendues au fil du funambule permet aussi de concevoir, au cœur du roman, la façon plus ou moins fortuite dont se croisent les trajectoires et destins respectifs du funambule et d'une poignée de New-Yorkais. Quand le funambule, après sa nuit d'amour, s'avance sur la corde que d'autres ont distendue en se l'appropriant, ses repères personnels se sont d'ailleurs modifiés: «He crawled out on the wire, which had grown loose and dangerous, but was still taut enough to hold him, and walked back in through his window» (239). Sur son fil alourdi par ces vies autres que la sienne qui s'y sont subrepticement suspendues, le funambule, dessinant une véritable poétique de l'équilibre, traverse la ville au petit matin, comme il le fera quelques semaines plus tard, cette fois au vu et au su de tant de gens qui, en ce 7 août 1974, laisseront entrer ce fil dans la trame de leur existence.

5 Car Let the Great World Spin est un roman choral : l'intrigue est multiple, fondée sur un nombre important de personnages dont le lecteur suit les trajectoires dans autant de chapitres dont ces personnages sont narrateurs et/ou focalisateurs internes; ces différents personnages se croisent de diverses façons au fil des pages (plus ou moins directement, plus ou moins consciemment) de telle sorte qu'à la fin du roman, le lecteur doit être capable de les placer dans une même constellation et comprendre la façon dont chaque planète évolue par rapport aux autres. Ce qui peut donc sembler de l'ordre du hasard et de la coïncidence, à la première lecture d'un tel roman, se trouve rétrospectivement élevé au rang de système. Dans Let the Great World Spin, un lieu (les tours jumelles du World Trade Center), une date (le 7 août 1974) et une heure (entre $7 \mathrm{~h}$ et $8 \mathrm{~h}$ du matin) suffisent à nouer les fils complexes de l'intrigue, la performance du funambule figurant le même point de mire pour tous les personnages. Qu'ils assistent à ce spectacle inattendu, qu'ils le suivent au téléphone depuis l'autre bout du pays, qu'ils en entendent parler rétrospectivement ou qu'ils en lisent un compte-rendu dans le journal, qu'ils soient émerveillés, indifférents ou même outrés devant l'audace de cet homme, tous les personnages du roman voient en effet l'ombre du funambule marchant dans les nuages se projeter, un instant au moins, sur leur existence.

6 Pour le lecteur, néanmoins, un niveau supplémentaire de convergence apparaît rapidement : bien que se situant en 1974, l'intrigue du roman est, en effet, habitée par les événements du 11 septembre 2001, les deux époques figurant tout d'abord la naissance des tours célébrée par Philippe Petit puis leur destruction simultanée dans l'attaque terroriste du 11 septembre. Tout un système d'échos est mis en place entre 1974, qui voit la fin de la guerre du Vietnam, la chute imminente de Nixon (sa démission intervient deux jours après la performance de Petit) et les débuts de ce qui deviendra l'internet (appelé arpanet à l'époque), et 2001, avec le début de la guerre contre le terrorisme, la montée en puissance d'une nouvelle présidence impériale et l'omniprésence des communications virtuelles. La lecture est donc double, le lecteur de 2009 ne pouvant s'empêcher de lire 2001 toujours déjà là dans cette histoire de 1974, ni de déchiffrer 1974 comme l'annonce de 2001. "Sometimes you have to go up to a very high floor to see what the past has done to the present » (306), dit l'un des personnages qui valide ainsi une lecture partagée entre 1974 et 2001. A la silhouette espiègle du funambule qui, en 1974, défie les fières profondeurs du canyon new-yorkais, répond d'ailleurs, en 2001, l'image de l'homme qui tombe sans fin le long des parois éventrées 
des tours, image immortalisée par la photographie iconique de Richard Drew, «The Falling Man », ou le roman éponyme de Don DeLillo (2007)ํ․

\section{Le funambule et l'homme qui tombe}

7 Or cet homme qui tombe, on le trouve aussi dans Let the Great World Spin, comme si le roman voulait également faire coïncider le funambule et l'homme qui chute au sens le plus propre du terme, en évoquant aussi directement que possible l'étymologie du mot latin, « coincidere » ou « tomber ensemble » : Corrigan, le moine irlandais installé dans un quartier mal famé du Bronx, est aussi charismatique que le funambule anonyme. Il côtoie lui aussi des abîmes, ceux de la prostitution, de la drogue et de la vieillesse, et dans ces bas-fonds, il resplendit comme une perle rare ( « the casts-off of New York the whores, the hustlers, the hopeless - all of those who were hanging on to him like he was some bright hallelujah in the shitbox of what the world really was ", 15). Ceux qui posent les yeux sur sa silhouette juvénile utilisent des comparaisons très similaires à celles que l'on trouve associées au funambule : un éternel enfant (« He was the type of child everyone smiled at. [...] People fell for him », 13-14), un ange (« he was, to them, a mad, impossible angel »,17) ou un acrobate à la recherche d'un improbable équilibre (son frère se souvient de lui, enfant - «balanced on top of the seawall, arms held wide » [12] - tandis que Tillie conclut: «Too old to be an acrobat, too young to die » [29]). Le roman tout entier le présente comme un homme qui s'épuise à porter les autres (« my younger brother who sparked people alive », 18), un moine qui vacille sous sa croix et que sa foi torture, un ange déchu (« he had fallen from grace »,58) qui meurt avec, dans ses yeux émerveillés, l'image du funambule qu'il a aperçu le matin même (" He had seen something beautiful, he whispered", 72).

8 Aussi, la structure de Let the Great World Spin n'est-elle pas simplement conçue autour du personnage du funambule; si celui-ci est indéniablement le clou du spectacle, l'axe autour duquel le vaste monde tourne pendant quelques heures, il partage néanmoins la page avec un jeune homme christique qui, à l'inverse, fracasse sa vie sur le bas-côté du Franklin Delano Roosevelt Drive. Le roman se développe donc autour de deux personnages, le funambule et Corrigan, qui peuvent apparaître comme une paire de jumeaux inversés : l'un, solitaire et entièrement dévoué à son art, aspire à s'élever audessus des hommes tandis que l'autre se débat au plus profond des bas-fonds newyorkais. Mais tous les deux sont engagés dans une quête mystique, que leurs pieds soient fermement posés sur un câble dans les nuages (« Walking was a divine delight. [...] He felt for a moment uncreated. Another kind of awake », 164) ou que leurs mains soient résolument plongées dans la laideur du monde ( «What Corrigan wanted was a fully believable God, one you could find in the grime of the everyday ", 20).

De ces deux silhouettes, découlent deux intrigues. Dans la première, celle que l'on pourrait baptiser l'intrigue "funambule», se déploie l'histoire des Soderberg, un couple aisé dont la vie se poursuit, dans leur appartement de Park Avenue, après la mort de leur fils au Vietnam. Claire, l'épouse dépressive, rencontre quatre autres mères de soldats disparus grâce à une petite annonce et, lors du brunch qu'elle organise chez elle afin de partager leurs peines, les cinq femmes en viennent à discuter de la performance du funambule que seulement l'une d'entre elles (Marcia) a vu le matin même depuis le ferry de Staten Island. Le juge Soderberg, lui, aura pour tâche de condamner le même funambule dans l'après-midi même de cette performance qu'il n'a 
pas vue, mais dont il perçoit, fasciné, l'audace et l'intelligence. Dans cette intrigue, il faut également inclure le groupe de hackers californiens qui assistent au spectacle du funambule par procuration, grâce à l'une de leurs nombreuses communications téléphoniques piratées; le lien qu'ils partagent avec les Soderberg s'établit grâce à Joshua, le fils Soberberg mort au Vietnam, qui était, lui aussi, un petit génie de l'informatique et travaillait sur les possibles utilisations de l'arpanet pour l'armée américaine. Enfin, on peut ajouter Tag, le gamin photographe qui prend le cliché du funambule figurant à la page $237 \mathrm{du}$ roman. La seconde intrigue, elle, l'intrigue "Corrigan", tourne autour du moine irlandais, de sa famille proche (Ciaran Corrigan, son frère, et Adelita, sa maîtresse) et de sa famille élargie (les prostituées du Bronx, et parmi elles Tillie et sa fille Jazzlyn, elle-même mère des petites Jaslyn et Janice). Lara, la jeune artiste dont l'amant est responsable de l'accident fatal de Corrigan sur le FDR, rejoint cette famille lorsqu'elle part vivre avec Ciaran.

\section{Une carte de New York : parallèles et intersections}

Lorsque l'on regarde de près le déroulement simultané des intrigues, on se rend compte que McCann place ses deux séries de personnages sur une carte de New York (où se déroule l'essentiel du roman), selon deux axes nord-sud qu'il dessine très clairement pour son lecteur, indiquant un nombre conséquent de points de repère (voir la carte en annexe à ce texte). L'intrigue "funambule », tout d'abord, s'égrène sur un axe dont la colonne vertébrale est dessinée par Park Avenue: l'appartement des Soderberg s'y trouve, et c'est aussi sur cette artère, un peu plus au nord (à l'intersection avec 116th street), que Gloria subit une agression qui la ramène vers Claire Soderberg et scelle leur amitié. Dans le prolongement sud de Park Avenue, l'on peut placer plusieurs points également liés aux Soderberg : le tribunal de Center Street, où exerce le juge, à côté de la prison, The Tombs, d'où sortent les criminels qui lui sont présentés, ainsi que Staten Island et sa ligne de ferry qui relie l'île à Manhattan, et qu'emprunte Marcia. L'axe "Corrigan », lui, se déploie de façon moins verticale depuis le sud du Bronx (où vivent le moine et les siens dans un quartier populaire proche du Concourse) jusqu'au sud de Manhattan, et son épine dorsale, plus sinueuse, est essentiellement composée par le Franklin Delano Roosevelt Drive qui longe la rive est de Manhattan, depuis Harlem jusqu'à Battery Park ; c'est sur cette voie à grande vitesse que Corrigan et Jazzlyn sont percutés par la voiture de Lara, et que se trouve également le Metropolitan Hospital où Corrigan est transporté et meurt sous les yeux de son frère et de sa maîtresse.

11 Si l'on poursuit cette étude topographique de Let the Great World Spin, on se rend compte que deux points de contact permettent à ces deux axes, a priori presque aussi verticaux et parallèles que les tours jumelles elles-mêmes, de coïncider. L'appartement des Soderberg, tout d'abord, est le premier lieu où les deux intrigues se croisent : Claire, de Park Avenue et Gloria, du Project du Bronx, s'y lient d'amitié le jour des événements et, le soir même, sont amenées, par le plus grand des hasards, à prendre en charge les deux orphelines de Jazzlyn. L'autre lieu qui permet aux personnages des deux intrigues parallèles de se croiser est le tribunal du juge Soderberg, puisqu'il accueille d'une part Tillie et Jazzlyn, accusées de cambriolage et soutenues par Corrigan et, d'autre part, le funambule que l'on vient d'arrêter pour être entré par effraction dans les tours et avoir organisé un spectacle illégal mettant en danger la vie d'autrui ; le juge traite les deux affaires dans la foulée, libère Jazzlyn qui se tuera quelques minutes plus tard avec 
Corrigan, condamne Tillie à huit mois de prison au cours desquels elle se suicidera, et invente pour le funambule une peine symbolique qui fait de lui le héros de la ville.

Le juge Soderberg est donc celui entre les mains duquel le destin des personnages-clefs se concentre un bref instant: il y a en effet, dans le tribunal du juge, intersection fortuite des deux intrigues mais, au-delà de cela, une coïncidence dans le regard qu'il porte sur les deux affaires, si différentes, qui lui sont présentées. Dans les deux cas, en effet, Soderberg rapporte les faits à l'échelle de la ville dont il estime avoir la charge et prononce une sentence qui, à ses yeux, fait sens dans cet univers qui en semble tant dépourvu. Soderberg, un conservateur qui soutient la guerre où son fils a pourtant trouvé la mort, porte tout d'abord un regard teinté de mépris sur le couple mère-fille qui comparait devant lui pour cambriolage ; ces prostituées, songe-t-il, font partie de cet infini défilé qui traverse son tribunal chaque jour, paumés, drogués, violeurs, travestis, amants violents et parents maltraitants, petits voleurs et grands mafiosi, tous habitants de ce New York chaotique et sauvage des années 1970. Depuis son estrade, Soderberg analyse cette ville qu'il connaît intimement :

[...] he wondered how the city had become such a disgusting thing on his watch. [...] the city burned down to the ground, got itself ready for its own little funeral of ashes, crime, crime, crime. There wasn't a bad thing in the city that didn't pass through Soderberg's gutter watch. (256-7)

Soderberg se considère comme le gardien de l'ordre précaire d'une véritable cour des miracles sur laquelle il rend une justice royale : la langue bien pendue de Tillie, qui ne le ménage pas, sa beauté défaite et sensuelle, qui le trouble, lui font, par exemple, prononcer une peine légèrement plus lourde qu'attendue en regard du crime commis. Certes, l'embrassade entre la mère et la fille, intense, l'émeut un instant, mais ne remet pas en question sa sévérité : n'est-il pas habitué à de telles démonstrations chez «ces gens-là »?

Still, it always surprised him, the love these people could display for each other. It was one of the few things that still thrilled him about the courtroom - the raw edge it gave to life, the sight of lovers embracing after beating each other up, or families glad to welcome back their son the petty thief, the surprise of forgiveness when it shone in the core of his court. It was rare, but it happened, and like everything, the rarity was necessary. (269)

Lorsqu'il choisit, par contre, de faire du funambule le fou du roi, énonçant pour lui un verdict à la fois clément et spirituel dont il aime à penser qu'il est à la hauteur de l'exploit du saltimbanque, Soderberg sait que l'éclat de l'événement rejaillira ainsi quelque peu sur lui. Dans ce cas précis, c'est également la connaissance que Soderberg a de la ville qui l'aide à formuler sa sentence : il n'a pas vu la performance du funambule mais il en comprend intuitivement le caractère exceptionnel, un peu à l'image du jeune Hegel qui, en 1806, voit passer Napoléon dans les rues d'Iéna. Clément Rosset s'attarde sur ce moment bref pendant lequel Hegel, «qui n'admet le réel que pour autant que celui-ci signifie » (42), est impressionné par « le porteur d'histoire » :

[...] c'est l'Histoire qui passe, qui se sensibilise un instant, sous les yeux du jeune Hegel, en la personne de Napoléon. Une histoire dont on ne saurait, dans l'immédiat, préciser le sens - car c'est la ruse de l'histoire, la ruse du sens, que ceux qu'ils concernent au premier chef la vivent et la produisent sans réussir à en pénétrer la signification. Aussi le cours des choses peut-il paraître anodin; et pourtant il manifeste le déroulement d'un sens. Apparemment il ne se passe rien de particulier ; en réalité, il se passe quelque chose. (43) 
Soderberg perçoit encore confusément que la traversée du funambule entrera dans l'histoire de la cité, et en déduit vite l'obligation qui est la sienne de rendre un jugement digne de l'événement. L'amende qu'il fixe est d'un penny par étage des tours gravies illégalement, soit $1,10 \$-$ un coup de génie, estime-t-il, digne de celui du funambule. La sentence de Solomon Soderberg le bien nommé trouve ainsi un équivalent urbain à l'acte du funambule et matérialise la réflexion ironique que se fait sa femme au sujet du même événement : «the intersection of a man with the city, the abruptly reformed, the newly appropriated public space, the city as art »(103), dit-elle de cette performance qu'elle n'a pas plus vue que son mari. Claire Soderberg, elle, exècre les tours jumelles pour la construction desquelles un vieux quartier a été détruit ; elle déteste leur arrogance et leur laideur, et les qualifie de "monstruosités » (95). Aussi la performance du funambule ne l'enchante nullement et se transforme, à ses yeux, en un acte intrusif et insupportable : l'homme, en effet, a pénétré les tours mais aussi son espace personnel, sa vie endeuillée par la mort de son fils. Les risques inouïs auxquels il soumet son corps, tout cela pour un petit numéro chaplinesque, lui sont intolérables parce qu'ils ôtent toute valeur à la vie de son propre fils mort au Vietnam : "How dare he do that with his own body?», se demande-t-elle avec colère. "Throwing his life in everyone's face? Making her own son's so cheap? Yes, he had intruded on her coffee morning like a hack on her code. With his hijinks above the city » (112).

16 Les époux Soderberg, points de croisement entre les deux intrigues, présentent donc deux points de vue radicalement différents sur cette coïncidence entre le funambule et la cité : pour le juge qui vient de se débarrasser des deux prostituées qui, à ses yeux, souillent la ville, le saltimbanque a trouvé un moyen flamboyant de célébrer New York par un acte seyant parfaitement à sa nature anhistorique et arrogante ${ }^{2}$; pour sa femme, il n'a fait que la violenter vulgairement et bouleverser ses habitants écorchés vifs. Et si Soderberg envoie sans états d'âme les prostituées vers un destin tragique, son épouse, qui n'en sait pourtant rien, rachète cette sévérité en prenant sous son aile Gloria, puis les fillettes orphelines.

\section{Multiplication de lignes matérielles et immatérielles}

17 Le lecteur comprend donc rapidement que le roman fonctionne sur tout un système d'intersections, de connections, de coïncidences qui lie les personnages les uns aux autres et qui impose un mode de lecture fondé sur la recherche d'indices et la reconnaissance des schémas. Parmi les images que McCann disperse au fil du roman, celle que le lecteur identifie le plus rapidement est d'ailleurs celle d'innombrables fils et lignes, autant d'échos au câble du funambule et d'incarnations du réseau de liens attachant les personnages les uns aux autres. Il semble difficile de les énumérer avec exhaustivité, mais on peut en mentionner quelques - uns parmi les plus structurels. Il y a, tout d'abord, les différentes lignes de transports urbains, si visibles sur la carte de New York précédemment utilisée, celles qui permettent les connections des boroughs de New York, telles que la mythique ligne de métro que prend Tag (premier chapitre du livre 2), qui part du Bronx, descend, entre cahots et pointes de vitesse, le long de Manhattan pour filer ensuite vers Brooklyn (ligne mythique qui fait l'ouverture magistrale de Libra, autre texte de DeLillo manifestement présent entre les lignes de McCann), ainsi que la ligne de ferry entre Staten Island et Manhattan (qui sera l'un des 
points de vue privilégié permettant une description de la performance du funambule), ou encore le Franklin Delano Roosevelt Drive mentionné plus haut. Mais on peut également penser à toutes ces lignes immatérielles sur lesquelles le roman insiste tant : les fils de l'arpanet, quasi magiques à l'époque, qui autorisent les premières communications virtuelles et composent ainsi la première toile dans ce qui ne s'appelle pas encore le cyberspace, qui repoussent les limites et font de Joshua Soderberg, par exemple, le "pilote d'une nouvelle frontière » (100) aux yeux fascinés de sa mère. Mais aussi le fil du téléphone, qui permet au Kid hacker de Californie de tomber amoureux de la voix d'une inconnue qui, depuis New York, lui décrit le spectacle du funambule; lorsque cette voix emploie le mot " fortuitous ", ce terme sonne si délicieusement à ses oreilles que le Kid panique à l'idée que cette femme découverte au hasard d'un de ses fréquents piratages de cabines téléphoniques, cette femme qui est son seul lien avec le spectacle du funambule mais qui est devenue bien plus pendant un bref instant, ne "s'évapore " sur la ligne (193). Ou encore les fils imaginaires, si saugrenus et pathétiques, auxquels se raccroche secrètement Claire Soderberg pour se sentir proche de son fils mobilisé : «It was as if she could travel through the electricity to see him. She could look at any electronic thing - television, radio, Solomon's shaver - and could find herself there, journeying along the raw voltage » (86). La nuit, Claire Soderberg s'assied donc près de son réfrigérateur, en ouvre parfois la porte, et voyage ainsi électriquement jusqu'au Vietnam, parle à son fils, lui caresse les cheveux... Il faut également évoquer les fils qui lient Corrigan aux machines qui tentent de le maintenir en vie à l'hôpital et que son frère ne peut quitter des yeux ( "I ntraveinous lines", 71 ; «My brother's heart machine blipping. The line like water», 71; « Heart monitoring flickering ", 72); ou encore les lignes de cocaïne qui structurent la vie de Lara, que son amant étale soigneusement sur son dos et qu'il aspire à même sa peau ( «I could feel the cocaine being sprinkled on my back [...] a rough line that he licked with his tongue ", 122), qu'elle ne peut s'empêcher de voir lorsqu'un avion passe dans le ciel et laisse un sillage blanc ( «A single jet stream cut across the sky, like a line of disappearing coke ", 127).

Chacun se raccroche à ces fils qui lui permettent d'être relié - à quoi ? A la vie, à la solitude d'un autre, à l'absence, au vide, à l'espace, à l'être ou au néant, à quiconque croisera son chemin à ce moment précis... «It's about being connected », affirme ainsi le Kid de Californie qui s'y connaît en matière de connections impalpables, avant de poursuivre « access, gateways, like a whispering game where if you get one thing wrong you've got to go all the way back to the beginning ", (197). Etre connecté, coïncider avec un autre, un inconnu, une voix, une présence virtuelle; et trouver un chemin, un passage, un code, avancer ainsi sur une route incertaine en mesurant son souffle... Tout au long de son roman McCann file deux métaphores, que le lecteur retrouve dans le sillage de la plupart des personnages.

\section{Lignes métaphoriques}

La première est initiée par l'image des chaussons du funambule, que McCann (dont on connaît la passion pour la danse ${ }^{3}$ ) décrit avec précision. Accessoires essentiels à son art, véritables outils de travail, ces chaussons font l'objet de tous les soins du jeune homme: en peau de buffle, noirs ( «dark-slippered foot », 7), ils sont si fins qu'ils permettent au câble de se « nicher» dans le creux du pied ( «The cable nested between his big and 
second toes for grip ", 163), et ainsi à tout le corps de ne faire plus qu'un avec le fil qui le soutient. D'emblée, ces chaussons semblent magiques: au moment même de sa performance, le funambule semble d'ailleurs savoir qu'ils seront au centre de l'image qu'il gardera de son exploit : "For years afterwards he'd still be up there : slippered, dark-footed, agile» (242). Or, le roman ne cesse d'insister également sur les pieds des autres personnages grâce à des gros-plans similaires, à commencer par Corrigan, bien sûr, alter-ego du funambule et doté, lui aussi, d'étranges chaussures. Grand lecteur de François d'Assise, Corrigan porte en effet des sandales qu'il a fabriquées lui-même ( « He made himself sandals out of some scrap leather and wore wild-colored socks underneath ", 21), dont son frère remarque l'extrême usure ( "The underside of his sandals were worn down. The sole had faded away, a little bubble of flat rubber », 41) et dont les prostituées se moquent avec tendresse ( «And those sandals! Man! We'd hear the slap of him coming», 225). A l'opposé, les filles qui l'entourent, elles, affichent toutes des talons aiguilles à la hauteur vertigineuse, et le texte ne laisse pas de mettre en valeur les stilettos jaune citron de Jazzlyn, dont un est retrouvé dans la camionnette accidentée aux côtés de la Bible de Corrigan (69), ou encore ceux, argentés, que sa mère porte au tribunal (266). Lors des funérailles de Jazzlyn, Lara remarque d'ailleurs la tenue décalée de toutes ces femmes rassemblées autour de la tombe d'un moine ( «the skirts too short, the heels too high ", 144). Enfin, les chaussures de Gloria sont à l'origine d'un incident qui infléchit de façon décisive le cours du roman. Ces chaussures sont en effet trop grandes et la blessent lorsqu'elle repart vers le Bronx, à pied, depuis l'appartement de Claire; volontairement choisies d'une demi-taille en dessus de sa pointure réelle pour lui permettre de les quitter facilement et ainsi de se sentir à l'aise lorsqu'elle va à l'opéra, son seul luxe, les chaussures de Gloria gênent sa marche comme celles qu'elle portait enfant, se souvient-elle, quand elle traversait les champs, dans le sud du pays, avec ses souliers achetés grands pour faire de l'usage, comprend le lecteur (307). Gloria chantonne un air de Nancy Sinatra ( «One of these days these boots are gonna walk all over you" , 307) pour oublier la morsure des chaussures. Plus encore que l'agression dont elle est victime, le lecteur a l'impression que ce sont bien ses pieds entaillés par le frottement des chaussures (qui en dit autant sur elle que ce que les stilletos révèlent des prostituées) qui brisent Gloria; ce sont ces talons à vif qui la ramènent vers Claire Soderberg, qu'elle avait pourtant fuie tant cette dernière lui semblait implorante et insistante ; ce sont encore eux qui incitent Claire à nettoyer et panser les plaies avec une sollicitude telle que Gloria redoute un instant de la voir lui sécher les pieds dans ses cheveux - image ironique qui permet néanmoins un retour aux sandales christiques de Corrigan, puis aux chaussons si souples du funambule lorsque Claire passe elle-même de consolantes pantoufles ( "slippers" , 310) aux pieds de cette femme forte que seul le Vietnam a réussi à abattre ( «It was Vietnam that brought me to my knees ", 313).

Tous ces personnages sont les arpenteurs quotidiens de New York, des «pratiquants ordinaires de la ville », pour reprendre l'expression de Michel de Certeau (141), et c'est bien ce que le texte nous dit, qui insiste tant sur leurs pieds plus ou moins bien équipés pour une telle aventure ; s'ils n'ont pas la grâce aérienne du marcheur céleste grâce à ses chaussons magiques, leur chemin individuel, qu'arpentent leurs chaussures toutes terrestres, est pourtant, lui aussi, aussi étroit qu'une corde raide. Le funambule a soustrait son corps «à l'emprise de la ville »: il «n'est plus enlacé par les rues qui le tournent et le retournent selon une loi anonyme » (Certeau 140), ce qui est le lot de ceux qui restent en bas, des marcheurs de Manhattan dont les pas, «au ras du 
sol » , «trament les lieux» (Certeau 147). Chacun, seul, trace sa route dans cette ville verticale, mais sur le fil horizontal de leurs pérégrinations, leurs pas se croisent et se font écho, comme le suggère d'ailleurs une réflexion de Gloria lors de sa longue et douloureuse marche vers le Bronx : «It had never occured to me before but everything in New York is built upon another thing, nothing is entirely by itself, each thing as strange as the last, and connected» (306). N'est-ce pas aussi ce que dit, à sa façon, Jaslyn, la petite orpheline élevée par Gloria avec l'aide de Claire, en repensant à un jeu qu'elle aimait pratiquer, enfant? Dans le dernier chapitre du roman, qui lui est consacré, elle se revoit courant à toute allure le long de la chaussée, un pied sur le trottoir et l'autre dans le caniveau, attentive à coordonner ses jambes ainsi décalées ; " an extended entrechat ", lui dit un jour Claire Soderberg, " half on, half off, half on, half off, half on « (330). Le double regard que portaient sur elle les deux femmes qui l'ont élevée, Gloria, noire et ronde dans sa robe à fleurs, et Claire, fine et élégante dans sa tenue de Park Avenue, semblait à Jaslyn, à l'époque, aussi boiteux et pourtant paradoxalement aussi harmonieux que son étrange course sur deux niveaux : "They looked so different, Claire in her neat skirt, Gloria in her flowered dress », pense-t-elle alors, « as if they too were running on different levels of pavement, but in the same body, the two of them combined» (330). Ce qui unit Claire et Gloria, ce sont les deux orphelines dont ces deux mères, tout aussi orphelines, acceptent la charge ; deux cœurs gros d'un même chagrin qui désormais vont l'amble, et battent de conserve.

21 Et l'on trouve là la seconde métaphore que McCann file tout au long de son roman, et qu'il rend particulièrement visible dans l'un des passages où l'on voit le funambule s'entraîner, au cœur de l'hiver, sur sa corde tendue en plein champ. Fasciné par la neige qui l'entoure ( «The whiteness thrilled him»), transporté par l'intensité de sa blancheur toute melvillienne ( «He was exuberant, almost stoned»), ébloui par une lumière que la neige transforme ( "The snow reinstructed the light, bent it, colored it, bounced it»), il fait un pas de côté qui manque lui être fatal; tombé droit dans l'épaisse couche de neige, il se retrouve alors piégé dans un sarcophage blanc: « He could feel the crystals on his ribcage, his navel, his chest », dit le texte (159) avant que le funambule ne parvienne à se hisser jusqu'à son câble et prenne la décision de ne plus jamais s'entraîner dans la neige. Cet instant d'inattention lui rappelle sa seule chute, des années auparavant, qui, cette fois, lui avait brisé des côtes ( «smashed several ribs », 161) ; depuis, chaque inspiration importante ravive cette douleur initiale - «like a tiny reminder, a prod near his hear » (161). Si cette menace de voir sa cage thoracique emprisonnée, écrasée et brisée, est clairement identifiée par le funambule, c'est-à-dire par celui qui, dans le roman, est le plus attentif à son corps, elle pèse également sur l'ensemble des personnages, à commencer par Corrigan, son double inversé. Corrigan, si maigre que ses côtes pointent sous sa chemise ( « and the bones of his ribcage were like some odd musical instrument», 41), si violemment projeté en avant lors de l'accident que le volant lui écrase le sternum ( «caught his chest and shattered his breastbone ", 69), dont l'état si désespéré pousse le chirurgien, à l'hôpital, à lui ouvrir ( «chest ripped open ", 70) la poitrine dont ne sortiront plus que quelques phrases murmurées et à peine audibles. Comme le funambule et Corrigan, nombreux sont les personnages qui sentent leur poitrine oppressée, et le mot «ribcage» ainsi que ses déclinaisons ( "ribs", "breastbone», «chest»...) abondent, le plus souvent en association avec une douleur émotionnelle. Claire Soderberg, posant les yeux sur une photo de son fils, sent par exemple comme une voix timide dans sa poitrine ( «She feels a little murmur at her ribcage ", 75), mais sait que la douleur n'est pas loin: «The 
simple things come back to us. They rest for a moment by our ribcages then suddenly reach in and twist our hearts a notch backward" (81). Lorsque Marcia, presque honteuse, confesse avoir pensé à un improbable retour de son fils en voyant le funambule, et avoue même avoir fui la scène sans vouloir savoir s'il était sain et sauf parce que s'il était vivant, cela signifierait qu'il n'était pas son fils, Claire est sans doute la seule à comprendre parfaitement cette phrase dans sa violente et absurde logique : «All of it like a slam in the chest » (99), résume-t-elle. Lorsque l'amant de Lara tente de la ramener vers lui après l'accident, c'est également sa poitrine tremblante qu'il enserre tandis que sa bouche parcourt la colonne vertébrale qu'il a saupoudrée de cocaïne ( His arm draped around my ribcage and his mouth at the center of my spine ", 122). Quant au juge Soderberg, il sait bien, lui, que c'est la ville qui, la première, broie en mille morceaux ces frêles poitrines dans lesquelles battent des chagrins trop violents, dont il égrène la liste dans une infinie litanie :

[...] he wondered how the city had become such a disgusting thing on his watch. How it lifted babies by the hair, and how it raped seventy-year-old women, and how it set fires to couches where lovers slept, and how it pocketed candy bars, and how it shattered ribcages, and how [...] (257)

La marche et le souffle : les deux métaphores filées tout au long du roman finissent par coïncider, elles aussi, pour les personnages de McCann, en ouvrant brièvement une fenêtre sur une vision d'une beauté insoutenable - et le terme n'est pas trop fort. Quand le funambule tombe dans la neige et sent ce corset de glace enserrer ses côtes, il lutte pour remonter sur son fil, parvient à s'agenouiller puis à s'allonger dessus, et reprend finalement sa marche. Comme le funambule sur son fil devenu sa ligne de vie, les personnages terrestres de Let the Great World Spin empruntent, le souffle court dans leurs poitrines serrées, les câbles et les fils que McCann a tracés pour eux sur la carte de New York, les avenues, les rues, les autoroutes, les lignes de métro et de ferry - autant de lignes de crête solitaires mais qui, sur la carte, forment elles aussi une fragile cage thoracique dans laquelle bat le cœur de la ville. Le funambule a retenu la leçon si douloureusement apprise dans le champ de neige: «Never again did he walk in the snow: he allowed that sort of beauty to remind him of what could happen » (160). Si la raison même de son art est la beauté d'une marche littéralement céleste ( "The core reason for it all was beauty. Walking was a divine delight », 164), l'extrême beauté, celle que recèle la couleur blanche, dont Melville disait qu'elle était «moins une couleur que l'absence visible de couleur, et en même temps le mélange de toutes", provoque néanmoins en lui l'effroi salvateur - n'est-ce pas ce qu'Ishmaël comprend, lui aussi qui, épouvanté par l'extrême blancheur du cachalot, tente d'expliquer sa terreur en évoquant un paysage hivernal semblable à celui dans lequel McCann place son héros : « is it for these reasons that there is such a dumb blankness, full of meaning, in a wide landscape of snows - a colorless, all - color of atheism from which we shrink? $»^{4}$ Le funambule se gardera bien, à l'avenir, de s'élancer sur son fil lorsque la neige immaculée scintillera autour de lui, ensorcelante de beauté. Corrigan, son malheureux jumeau, a-t-il su se protéger de cette puissante magie ? Sur son lit d'hôpital, le moine dont la camionnette est sortie de l'axe du FDR Drive, ne songe qu'à la chose magnifique dont il a été le témoin, à cette « chose de beauté » proprement keatsienne :

He was saying something in his morphine haze. He had seen something beautiful, he whispered. [...] Corrigan whispered something to her again, the blood bubbling at his mouth. I touched her forearm. What's he saying? Nonsense, she said, he's talking nonsense. He's hallucinating. Her ear to his mouth now. Does he want a 
priest? Is that what he wants? She turned to me. He says he saw something beautiful. (72)

C'est l'image merveilleuse d'un homme qui marche dans les nuages, comme imprimée sur sa rétine, qui accompagne Corrigan dans son agonie, cette image qui date du petit matin, que l'accident n'a pu effacer, et qui se substitue au réconfort éventuel de sa religion. Gloria, elle aussi, le sait bien, qui confie sa sagesse à ses deux filles, à la veille de sa mort: «The only thing worth grieving over, she said, was that sometimes there was more beauty in this life than the world could bear " (339). Dans ce roman où tant de choses se répondent, se dédoublent, s'entrecroisent, se superposent, se synchronisent, l'épiphanie, moment où coexistent terreur et bonheur mêlés, naît précisément d'un instant où le vaste monde ne coïncide plus tout à fait avec lui-même parce que la vie contient soudain un surplus de beauté dont, désemparé, il ne sait que faire.

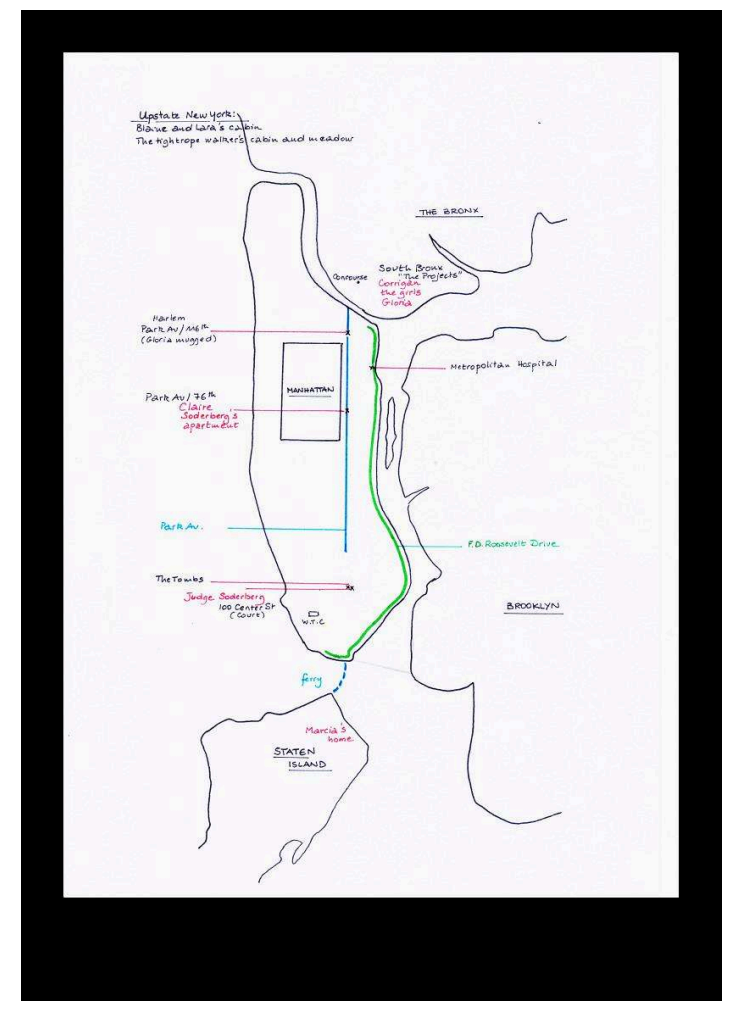

Let the Great World Spin : carte de New York, axes et points principaux mentionnés dans les intrigues (Sophie Vallas).

\section{BIBLIOGRAPHIE}

Certeau (de), Michel. « Marches dans la ville », L'invention du quotidien. 1. Arts de faire. Paris: Gallimard, 1990.

DeLillo, Don. Libra. New York: Viking, 1988. 
---. Falling Man. New York: Simon and Schuster, 2007.

Kachka, Boris. « Novelist Colum McCann on Let the Great World Spin and the 9/11 'Grief Machine'", 6/23/09, HYPERLINK ».

McCann, Colum. Dancer. Londres: Weidenfeld, 2003.

---. Let the Great World Spin. London: Bloomsbury, 2009.

Melville, Herman. Moby - Dick, or The Whale [1851]. Penguin Classics, 1972.

---. Moby - Dick, ou le Cachalot. Trad. Philippe Jaworski, Paris : Gallimard (La Pléiade), 2006.

Rosset, Clément. Le Réel. Traité de l'idiotie. Paris : Minuit, 1972/2004.

Iconographie

Drew, Richard. « The Falling Man », Associated Press, 9/11/2001.

Marsh, James (dir.). Man on Wire. Wall to Wall Production, 2008.

\section{NOTES DE FIN}

1. Dans un entretien avec Boris Kachka, McCann a confié avoir longtemps pensé que la première phrase de son roman serait: "The prospect of a falling man ». Lorsque le roman de DeLillo est sorti, il a renoncé à sa phrase d'ouverture, mais l'image de l'homme qui tombe traverse bien entendu tout le roman.

2. " There was an arrogance in it, he knew, but on the wire the arrogance became survival ", 240.

" The Twin Towers. Of all places. So brash. So glassy. So forward-looking " 248.

3. Voir son roman paru en 2003, Dancer.

4. Herman Melville, Moby-Dick, chapitre 42, " The Whiteness of the Whale " (296). Le passage en français est emprunté à la traduction de Philippe Jaworski (224).

\section{RÉSUMÉS}

Dans Let the Great World Spin (2009), la coïncidence est élevée au rang de système : roman choral, l'ouvrage de Colum McCann construit savamment un réseau d'intrigues, de reflets et d'échos au centre duquel se trouve la performance d'un funambule qui, le 7 août 1974 au matin, marche sur un câble tendu entre les deux tours jumelles du World Trade Center à peine érigé. Toute lecture de Let the Great World Spin ne peut que faire coïncider la naissance des tours ainsi célébrée par le funambule et leur destruction en 2001, d'autant que McCann crée, au cœur de son texte, la figure d'un homme qui tombe, reflet inversé de celle de l'acrobate. Une carte de New York se dessine au fur et à mesure du roman, sur laquelle l'ombre de l'intrigue aérienne se projette, se mêlant aux déplacements tout terrestres des personnages accablés par de bien lourdes vies : les silhouettes se meuvent le long de deux axes aussi verticaux et parallèles que les tours jumelles elles-mêmes et se croisent au hasard de points de contact et d'intersection, autant d'occasions pour ces destinées solitaires de réfléchir le moment magique pendant lequel un homme a tracé un trait d'union dans le skyline de New York.

In Let the Great World Spin coincidence is structural: the choral novel rests on a complex network of plots, echoes and reflections hinging on the performance of a tightrope walker who, on 7 August 1974, crosses from the top of one of the brand new Twin Towers to the other. Reading Let the Great World Spin, one is bound to superimpose the birth of the towers in 1974, which the 
tightrope walker celebrates, and their destruction in 2001, especially since McCann creates the haunting figure of a falling man at the very heart of his text, as a mirrored and reversed image of the skywalking acrobat. Little by little a map of New York appears, on which the shadow of the upper and cloudy plot adds to the various wanderings of the down-to-earth characters burdened with their painful existence. The characters move along two vertical and parallel lines which recall the towers themselves, and their destinies intersect in different points, thus reflecting this magical hour during which a man's cloudwalk managed to draw a hyphen in the city's skyline.

\section{INDEX}

Keywords : Let the Great World Spin, 9/11, choral novel, coincidence, New York, map

Mots-clés : Let the Great World Spin, 11 septembre, roman choral, coincidence, New York, carte

\section{AUTEURS}

\section{SOPHIE VALLAS}

Aix-Marseille Université (LERMA, EA 853)

Professeur de littérature américaine

sophie.vallas@univ-amu.fr 